\title{
Rôle de l'action collective et du capital social dans les processus d'adaptation au changement climatique en zone côtière - Comparaison de deux études de cas au Nouveau-Brunswick (Canada)
} Role of collective action and social capital in the process of adaptation to climate change in coastal areas - Comparison of two case studies in New Brunswick (Canada)

Mélinda Noblet, Julie Guillemot et Omer Chouinard

\section{(2) OpenEdition}

Édition électronique

URL : http://journals.openedition.org/developpementdurable/11297

DOI : 10.4000/developpementdurable. 11297

ISSN : 1772-9971

Éditeur

Association DD\&T

Référence électronique

Mélinda Noblet, Julie Guillemot et Omer Chouinard, « Rôle de l'action collective et du capital social dans les processus d'adaptation au changement climatique en zone côtière - Comparaison de deux études de cas au Nouveau-Brunswick (Canada) », Développement durable et territoires [En ligne], Vol. 7, n² | Juillet 2016, mis en ligne le 28 juillet 2016, consulté le 01 mai 2019. URL : http://

journals.openedition.org/developpementdurable/11297; DOI : 10.4000/developpementdurable.11297

Ce document a été généré automatiquement le 1 mai 2019.

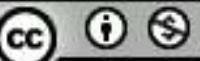

Développement Durable et Territoires est mis à disposition selon les termes de la licence Creative Commons Attribution - Pas d'Utilisation Commerciale 4.0 International. 


\section{Rôle de l'action collective et du capital social dans les processus d'adaptation au changement climatique en zone côtière - Comparaison de deux études de cas au Nouveau-Brunswick (Canada)}

Role of collective action and social capital in the process of adaptation to climate change in coastal areas - Comparison of two case studies in New Brunswick (Canada)

Mélinda Noblet, Julie Guillemot et Omer Chouinard

1 L'adaptation a longtemps été pensée par la communauté internationale comme étant fondée sur un processus décisionnel Top Down. Mais, depuis une dizaine d'années, plusieurs auteurs estiment à juste titre que cette approche est limitée, il apparait de plus en plus évident pour la communauté scientifique que l'adaptation est avant tout un processus local . Pour autant, l'échelle locale est-elle en mesure de gérer l'adaptation ? En s'intéressant au rôle de l'action collective et du capital social dans les processus d'adaptation de communautés côtière situées au Nord et au Sud du Nouveau-Brunswick, cet article met en évidence les limites et les leviers à l'adaptation à l'échelle locale. Nous verrons dans quelles mesures les problèmes posés par les impacts du changement climatique (CC) suscitent ou non une prise en charge collective à l'échelle locale, profitant ou non au renforcement des capacités d'adaptation.

2 L'adaptation est définie et abordée de différentes manières au sein de la littérature scientifique. Dans le cadre de cette recherche, nous comprenons l'adaptation comme étant un processus impliquant des changements dans les systèmes socio-écologiques en 
réponse aux impacts du $\mathrm{CC}$ vécus et/ou anticipés. Ces réponses peuvent viser le court ou le long terme et avoir des objectifs strictement liés aux impacts du CC ou intégrer de nombreuses autres dimensions, dans une perspective de transformation du système (adapté de Moser et Ekstrom, 2010). Par ailleurs, il est courant de distinguer « adaptation planifiée » et "adaptation autonome». Nous avons choisi de nous concentrer sur l'adaptation autonome tout en considérant les liens qui peuvent s'établir entre adaptation autonome et adaptation planifiée. En effet, nous estimons que l'adaptation relève autant du secteur public que du secteur privé. L'adaptation autonome est comprise ici comme étant le résultat de l'action portée par des individus, des groupes, des associations, des entreprises, etc. et menée à l'échelle locale sans intervention directe de l'autorité publique (Noblet, 2015). Elle peut aussi bien être individuelle que collective, anticipée que réactive.

Dans cette perspective, nous allons nous intéresser au lien entre capital social et action collective: dans le cadre des approches collectives d'adaptation observées dans les communautés côtières néo-brunswickoises, quelle forme de capital social est sollicitée ? Quels sont les acteurs mobilisés et surtout les liens activés? À travers une approche comparative, nous souhaitons mettre en évidence les divergences et convergences observées entre les deux sites à l'étude et ainsi contribuer plus largement à la réflexion sur la portée mais aussi les limites de l'adaptation à l'échelle locale.

\section{Cadre théorique et conceptuel}

\subsection{Le capital social : un concept populaire}

De la sociologie où il est analysé pour la première fois, à l'écologie politique en passant par l'économie, les études du développement, les sciences de l'environnement, le concept de capital social s'est largement popularisé depuis les années 1980. Selon A. Portes (1998), la première analyse contemporaine du concept de capital social a été produite par $\mathrm{P}$. Bourdieu au cours des années 1970-1980 dans le cadre de sa production théorique consacrée à l'espace social. Ce dernier définit le capital social comme étant «l'ensemble des ressources actuelles ou potentielles qui sont liées à la possession d'un réseau durable de relations plus ou moins institutionnalisées d'interconnaissance et d'interreconnaissance » (Bourdieu, $1980:$ 2). Du côté nord-américain, le concept de capital social sera popularisé par les travaux de J. Coleman. Ce dernier s'intéresse plus particulièrement à l'analyse structurelle des interactions existant entre les individus et facilitant l'action collective (Coleman, 1988).

5 Dans les années 1990, R. D. Putnam va renforcer la popularité du concept de capital social. Dans ses travaux portant sur les collectivités en Italie, il met en évidence que les collectivités les plus efficaces sont celles qui «jouissent de réseaux d'activité communautaires élaborés : les gens établissent des liens entre eux et le gouvernement » (Putnam, 1999). Pour R. D. Putnam, le capital social correspond aux caractéristiques de l'organisation sociale telles que les réseaux, les normes et la confiance qui peuvent améliorer l'efficacité des sociétés en facilitant les actions coordonnées (Putnam et al., 1994). Les travaux de R. Putnam vont servir de base à l'intérêt porté par l'économie du développement au concept de capital social dans les années 1990. Contrairement aux premiers penseurs du concept (P. Bourdieu et J. Coleman), pour qui le capital social était 
perçu comme un facteur de promotion individuelle, le capital social est perçu par les économistes du développement comme un facteur de développement des communautés.

\subsection{Capital social, action collective et changements environnementaux}

Dans le cadre des études portant sur la gestion des ressources naturelles et les changements environnementaux, de nombreux auteurs (E. Ostrom, 1990, 1996; F. Berkes et $C$. Folke, 1998) font appel au concept de capital social qu'ils utilisent pour se référer à la richesse de l'organisation sociale (Ostrom, 2008). Le capital social forme la base d'une action collective organisée et est indispensable à la gouvernance des ressources communes. Depuis le début des années 2000, le concept fait son apparition dans le cadre des recherches portant sur l'adaptation au changement climatique .

7 W. N. Adger et al. (2003) estiment que l'adaptation dépend en grande partie de la capacité des communautés à agir collectivement. Les travaux de W. N. Adger (2003) sur le capital social s'inscrivent dans l'approche développée par les économistes du développement pour qui le capital social est un facteur de développement des communautés ${ }^{1}$, en considérant que dans le cadre du changement climatique le capital social est un facteur d'adaptation: "a necessary 'glue' for adaptive capacity, particularly in dealing with unforeseen and periodic hazardous events" (Adger, 2003, p. 392).

Pour W. N. Adger, le processus d'adaptation implique "the interdependence of agents through their relationships with each other, with the institutions in which they reside, and the resource base on which they depend" (Adger, 2003, p. 400). Ainsi, la constitution d'un capital social favorisant, à priori, une action collective d'adaptation ne concerne pas uniquement les acteurs situés à l'échelle locale en relation avec des ressources territoriales spécifiques, elle s'étend également aux relations entre les citoyens, organisés ou non en collectif, et l'État. En effet, la capacité des individus à agir dans leur intérêt collectif dépend de la qualité des institutions formelles (Adger, 2003) et du soutien que ces dernières offrent au développement et au maintien du capital social. Nous savons que l'adaptation au changement climatique nécessite l'intervention des pouvoirs publics pour un certain nombre d'actions (construction de digues en bord de mer, évaluation des zones à risques, etc.) auxquelles l'action collective autonome ne peut se substituer, mais, elle peut y contribuer ou même participer à sa co-production. D'après E. Ostrom (1996), l'implication des citoyens dans la résolution de problèmes publics et dans la mise en œuvre des solutions peut nécessiter plus de temps à l'étape initiale d'un processus, mais promettre, de meilleurs résultats à long terme.

9 Toutefois, il est important de mentionner que plusieurs recherches avancent que les relations de confiance et de réciprocité ne favorisent pas toujours l'émergence des actions collectives ou individuelles attendues (Callois, 2006; Wolf et al., 2010). Ces relations peuvent notamment amplifier la résistance au changement et favoriser l'adoption de pratiques augmentant la vulnérabilité des communautés. Nous verrons à travers les études de cas que la nature du capital social sollicité peut effectivement affecter le processus d'adaptation. 


\section{Contexte des recherches et méthodologie}

\subsection{Présentation des sites d'étude}

\subsubsection{Caractéristiques physique des territoires étudiés et impacts du changement climatique}

Les sites de recherche sont situés au nord-est (Péninsule acadienne) et au sud-est (secteur de Cocagne situé à proximité de Moncton)du Nouveau-Brunswick (N.-B.) (carte cidessous). Il s'agit de régions côtières, traditionnellement tournées vers l'exploitation des ressources naturelles, dont les pêches. Le tourisme est en développement, notamment au sud-est du territoire. Le littoral du N-B est particulièrement sensible aux effets du CC : environ $70 \%$ de la côte de la province est en érosion(Environnement Canada, 2006) et plusieurs communautés, du fait de la faible élévation de leur territoire par rapport au niveau de la mer, ont déjà subi des inondations importantes lors d'épisodes de submersion marine (Guillemot et al., 2013 ; Chouinard et al., 2012). Ces phénomènes auront tendance à s'intensifier avec l'augmentation des températures et l'élévation du niveau marin (Daigle, 2014; Aubé, 2014). En effet, les pronostics d'élévation du niveau marin pour le siècle prochain (2000-2100) se situent entre 50 et $60 \mathrm{~cm}+-35 \mathrm{~cm}$ (Environnement Canada, 2006). Par ailleurs, une diminution du couvert de glace est déjà observée (Stervinou, 2013). Le modèle climatique canadien indique que d'ici 2050 le Golfe du SaintLaurent sera libre de glace (Environnement Canada, 2006). Il est reconnu par les scientifiques que la présence de glace a un effet modérateur sur l'impact des tempêtes (Robichaud et al., 2012). Ainsi, la réduction du couvert de glace entraine une exposition plus importantes des côtes aux tempêtes hivernales ainsi qu'un renforcement de l'érosion (Bernatchez et al., 2008).De nombreux impacts sont déjà observés sur le territoire : la disparition de plages, la destruction de routes côtières et d'infrastructures comme des quais de pêche, plusieurs habitations sont aussi menacées. Les impacts des changements climatiques sont également susceptibles d'avoir des effets sur plusieurs activités d'exploitation des ressources naturelles comme l'extraction de tourbe, la pêche, l'agriculture ou le tourisme (Environnement Canada, 2006). 


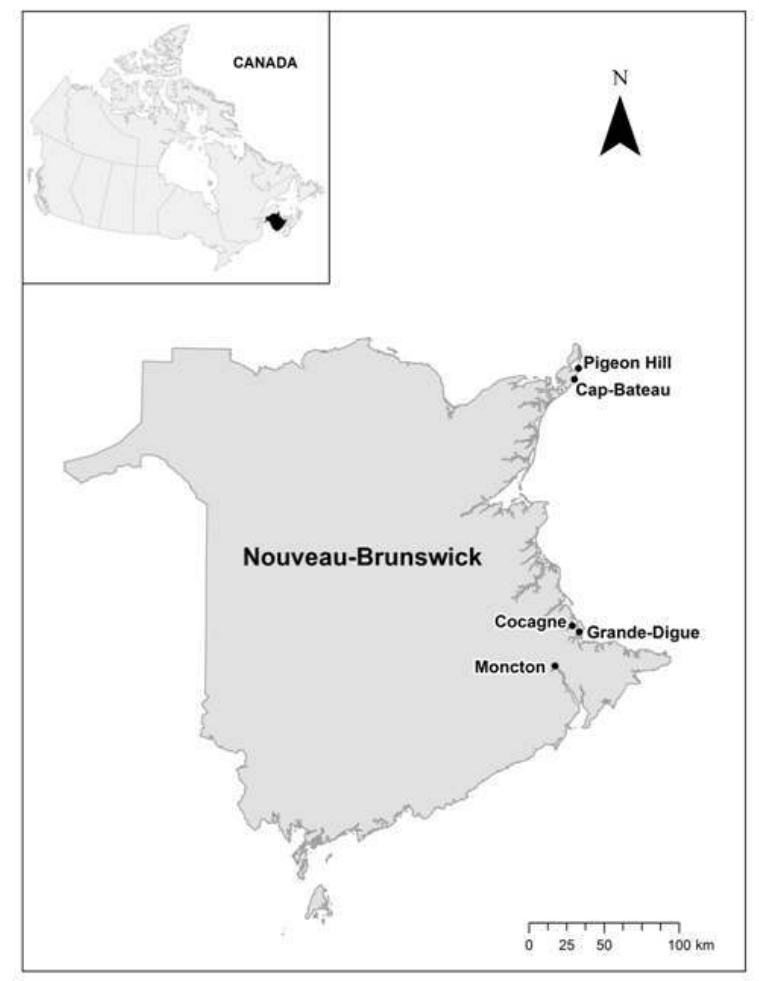

\subsubsection{La singularité du Nouveau-Brunswick en matière de gouvernance locale}

Pour mieux comprendre, les enjeux propres au Nouveau-Brunswick, il est indispensable de rappeler rapidement la particularité du système de gouvernance locale de cette province. En effet, seulement $30 \%$ du territoire est municipalisé, les $70 \%$ restant, appelés Districts de Services Locaux (DSL), sont directement administrés par le gouvernement provincial. Une portion importante de ces territoires est non habitée(terres publiques) ou très peu peuplée. Cependant sur une population néo-brunswickoise totale de 750000 personnes, environ $35 \%$ vivent dans un DSL et ne sont donc pas représentées au niveau local par une autorité élue (Finn, 2008). Les habitants des DSL peuvent se doter d'un comité consultatif de trois à cinq membres, mais ce dernier n'a aucun pouvoir administratif, financier ou contractuel. Considéré par le gouvernement provincial comme étant une forme de gouvernance locale (Finn, 2008), le DSL constitue dans les faits une simple entité déconcentrée du gouvernement provincial (Noblet, 2015). Plusieurs localités ne sont même pas représentées localement par un comité faute d'intérêt de la part de la population. Tous les sites étudiés dans le cadre de cette recherche sont, au moment de l'enquête, des DSL ce qui limite la possibilité d'intervention d'acteurs publics locaux, que cela soit en soutien au développement, à la planification des usages ou dans le cadre de la gestion des risques. 
Tableau 1. Types de gouvernance locale au Nouveau-Brunswick

\begin{tabular}{|c|c|c|c|}
\hline \multirow[b]{2}{*}{$\begin{array}{l}\text { Type de structure de } \\
\text { gouvernance locale }\end{array}$} & \multirow[b]{2}{*}{ Représentation locale } & \multicolumn{2}{|c|}{ Services aux citoyens } \\
\hline & & $\begin{array}{l}\text { Services fournis par la } \\
\text { structure locale }\end{array}$ & $\begin{array}{l}\text { Services fourn is par le } \\
\text { gouvernement } \\
\text { provincial }\end{array}$ \\
\hline $\begin{array}{c}\text { Municipalité (cité, } \\
\text { ville, village) } \\
(101)\end{array}$ & Conseil élu pour 4 ans & $\begin{array}{l}\text { Administration, } \\
\text { aménagement du } \\
\text { territoire, services } \\
\text { d'urgence, services de } \\
\text { police, routes, } \\
\text { collectes des ordures }\end{array}$ & \\
\hline $\begin{array}{l}\text { Communauté rurale } \\
\text { (6) }\end{array}$ & Conseil élu pour 4 an & $\begin{array}{l}\text { Administration, } \\
\text { urbanisme et urgence }\end{array}$ & $\begin{array}{l}\text { Services de police, } \\
\text { routes, collectes des } \\
\text { ordures, etc. }\end{array}$ \\
\hline $\begin{array}{l}\text { District de service } \\
\text { local (265) }\end{array}$ & $\begin{array}{l}\text { Comité consultatif } \\
\text { Pas de pouvoir de } \\
\text { décision } \\
\text { Administré et géré par } \\
\text { le gouvernement } \\
\text { provincial }\end{array}$ & & $\begin{array}{l}\text { Tous les services sont } \\
\text { fournis par le } \\
\text { gouvernement } \\
\text { provincial }\end{array}$ \\
\hline
\end{tabular}

\subsubsection{Description des territoires}

\section{Cap Bateau et Pigeon Hill (nord de la province)}

12 Au nord-est du Nouveau-Brunswick, nous avons travaillé auprès des communautés de Cap-Bateau ( $\mathrm{CB}$ ) et Pigeon Hill (PH) situées à l'est de l'île Lamèque (carte ci-dessus). Un processus de municipalisation du territoire visant à regrouper tous les DSL et les deux municipalités des îles Lamèque et Miscou est en cours, mais plusieurs ressortissant des DSL étudiés s'y sont opposés à travers une pétition mise en circulation en $2014^{2}$.Sur le plan géomorphologique, cette zone est généralement composée d'une alternance de plages et de falaises friables d'une hauteur de 2 à $5 \mathrm{~m}$, directement exposées aux assauts $\mathrm{du}$ Golfe du Saint-Laurent. L'érosion constitue un problème majeur pour ces communautés, dans ce secteur des taux d'érosion variant de $30 \mathrm{~cm}$ à $1,2 \mathrm{~m}$ par an y sont répertoriés pour la période 1974-2009 . La principale activité économique est encore aujourd'hui la pêche, même si cette activité est moins florissante que par le passé. Sur le plan démographique, les deux villages sont faiblement peuplés (CB : 276 habitants, 2011 et PH : 489 habitants, 2011), la villégiature y est encore peu développée probablement à cause de l'isolement de ces communautés, et les résidents sont souvent ancrés dans ces villages, voire dans leurs habitations, depuis plusieurs générations ${ }^{3}$. Dans ce secteur, le niveau d'éducation est plus faible que dans l'ensemble de la province ainsi que le revenu moyen des ménages (Coup d'œil sur ma région ${ }^{4}$ ).

\section{Cocagne et Grande-Digue (sud-est de la province)}

13 Au sud-est de la province, nos recherches ont été menées au sein des communautés de Cocagne et Grande Digue situées sur le front de mer, face au Détroit de Northumberland (carte ci-dessus). Ces dernières bénéficient de la proximité de la ville de Moncton qui se trouve à environ quarante kilomètres. Elles sont davantage peuplées et urbanisées que les communautés situées au Nord (Cocagne : 2545 habitants, 2011 et Grande-Digue : 2182 habitants, 2011), même si, comme au Nord la population y est décroissante et 
vieillissante. Tout comme les communautés de la Péninsule acadienne, les villages de Cocagne et Grande-Digue sont traditionnellement tournés vers la mer, du fait des activités de pêche auxquelles se sont ajoutés ces dernières décennies le tourisme et la villégiature. Sur le plan géomorphologique, ce territoire est caractérisé par un relief bas reposant sur un substrat gréseux et friable (Environnement Canada, 2006). Les communautés étudiées subissent principalement des dommages d'inondation, liée aux ondes de tempêtes, et plusieurs plages sont en érosion. Au moment de notre enquête, les deux communautés étaient des DSL. Depuis 2014, Cocagne est devenue une communauté rurale tandis que Grande-Digue s'interroge encore sur l'évolution à donner à sa communauté. Enfin, il est à noter que les revenus des particuliers sont généralement plus élevés au Sud qu'au Nord ${ }^{5}$.

\subsection{Contexte de la recherche et méthodologie}

Cette recherche est de nature qualitative. Nous avons utilisé les résultats de l'analyse thématique d'une série d'entrevues semi-dirigées réalisées en 2012 au sein de communautés côtières affectées par les impacts des CC.

La recherche menée dans le Sud-est s'est inscrite dans le cadre d'une enquête de terrain de type ethnographique (observations participantes, entrevues, carnet de terrain, etc.) menée sur une période de 4 mois en $2012^{6}$.Les résultats présentés dans cet article reposent sur l'analyse de 20 entrevues semi-dirigées ${ }^{7}$ et d'un groupe de discussion ${ }^{8}$ réalisés au sein des communautés de Grande-Digue et Cocagne.

16 Au nord-est la collecte de données s'est déroulée dans le cadre d'un programme de recherche partenariale (Fontan, 2010) portant sur l'accompagnement de communautés côtières affectées par le changement climatique (Alliance de Recherche Université Communauté-Défis des Communautés Côtières). Nous avons utilisé les données issues de l'analyse de 10 entrevues semi-dirigées faites auprès d'acteurs des villages de Pigeon Hill et Cap-Bateau', de 20questionnaires soumis aux résidents de Cap-Bateau et des notes de terrain prises lors des réunions organisées de 2012 à 2014 dans le cadre de ce projet.

\section{Actions individuelles et collectives d'adaptation : contrastes observés entre les deux zones d'étude}

\subsection{Réactions individuelles : protection ou déplacement face à la menace}

Plusieurs répondants considèrent les impacts potentiels du CC sur des activités sectorielles : la pêche dans la Péninsule acadienne et l'agriculture dans le sud-est. Dans le cadre de cet article, nous avons choisi de traiter davantage des impacts associés aux constructions en zone côtière. En effet, la menace la plus vive pour les communautés étudiées concerne leurs propriétés et le maintien de leur mode de vie sur le littoral. Au sud-est, le fait de vivre en face de la mer est synonyme de réussite sociale et de bien-être, l'aboutissement d'une vie réussie pour nombre de retraités. Pour certains, c'est une vie d'économies qu'ils ont investi dans leur propriété de bord de mer, il n'est donc pas question de laisser partir son terrain à la mer. Dans les villages de $\mathrm{CB}$ et $\mathrm{PH}$, les propriétaires tiennent aussi à leurs maisons face à la mer, non seulement à cause du cadre 
et des investissements qu'ils y ont fait, mais aussi parce pour plusieurs il s'agit de leur lieu de vie depuis la naissance ${ }^{10}$. Afin de conserver leurs biens, nous avons observé que les propriétaires concernés mettent en œuvre deux options principales : la protection et le déplacement.

Tout d'abord, la réaction la plus visible et la plus généralisée est la protection contre l'érosion et l'impact des tempêtes, à travers la mise en place de plusieurs types de techniques de défense côtière : enrochement, digue en bois, en béton, utilisation de pneus usagés, etc. Comme le souligne un résident retraité dont la maison se situe à moins de 100 $m$ de la mer, "l'adaptation c'est ce qu'on fait naturellement pour se protéger » et estime que sa propriété n'est pas à risque car «les gens (ceux qui habitent devant chez lui, tout au bord de l'eau) ont protégé la côte » (Répondant Grande Digue, 4 juin 2012). Une autre résidente explique que depuis les tempêtes de 2010 «les gens continuent de hausser les murs, d'ajouter des roches, ils se préparent" (Répondant Cocagne, 24 mai 2012). Cette "protection du littoral » est plus courante dans le sud-est où la moindre élévation du relief et les moyens financiers des propriétaires la rend plus accessible. Les habitants des zones submersibles apprennent aussi à s'accommoder des effets sporadiques des tempêtes : inondations de sous-sols et des fosses septiques, salinisation des puits artésiens etc. Ils peuvent, dans certains cas, se qualifier pour un remboursement des dégâts dans le cadre du programme d'aide post-tempête du gouvernement (Hébert, 2015).

19 Par ailleurs, à PH et CB plusieurs habitations initialement construites sur les falaises au cours de la colonisation du territoire par les familles de pêcheurs sont maintenant menacées. Certaines ont déjà été déplacées en retrait au frais de leur propriétaire ${ }^{11}$, puisqu'il n'existe aucun programme gouvernemental pour cette option. Une douzaine d'autres sont menacées à court terme et leur déplacement est envisagé (Hébert, 2015) .Les résultats de la recherche mettent également en évidence que le déplacement n'est pas une option envisagée dans le sud-est. Malgré le recours au déplacement dans le secteur du nord-est, il faut noter que l'option de la protection est préférée par les propriétaires tout comme dans le sud-est, mais dans ce secteur cette option est beaucoup moins abordable et donc très peu appliquée par les propriétaires.

Ces actions sont majoritairement portées par des individus, mais confrontées aux limites de ces approches individuelles, notamment le manque de ressource de certains individus mais aussi la nécessité de mieux coordonner les actions individuelles pour éviter les conflits et anticiper le changement, les acteurs se mobilisent dans le cadre de démarches collectives. 
Tableau 2. Présentation des approches collectives et individuelles d'adaptation en lien avec le capital social mobilisé

\begin{tabular}{|c|c|c|}
\hline \multicolumn{3}{|c|}{ Adaptation au plan individuel } \\
\hline & Nord-est de la province & Sud-est de la province \\
\hline $\begin{array}{l}\text { Action individuelle : } \\
\text { réduction du risque et } \\
\text { maintien du mode de vie }\end{array}$ & $\begin{array}{l}\text { - Déplacement des maisons } \\
\text { Protection des maisons : mesure } \\
\text { rare par manque de moyen } \\
\text { financier } \\
\text { - Entraide après les tempêtes }\end{array}$ & $\begin{array}{c}\text { Protection des maisons: } \\
\text { mesure majoritaire } \\
\text { Entraide après les tempêtes }\end{array}$ \\
\hline $\begin{array}{c}\text { Capital social sollicité : } \\
\text { Réseau } \\
\text { d'interconnaissance } \\
\text { individuel }\end{array}$ & \multicolumn{2}{|c|}{ - Réseau d'entraide : voisinage, famille, parenté, amis } \\
\hline \multicolumn{3}{|c|}{ Adaptation au plan collectif } \\
\hline & Nord-est de la province & Sud-est de la province \\
\hline Action collective & $\begin{array}{l}\text { Développement d'un argumentaire } \\
\text { pour obtenir un soutien financier } \\
\text { et technique du gouvernement } \\
\text { pour protéger les maisons les plus } \\
\text { à risque permettant de faire face à } \\
\text { un danger immédiat (PH) }\end{array}$ & $\begin{array}{l}\text { Élaboration d'un plan local } \\
\text { d'adaptation } \\
\text { Action de sensibilisation sur le } \\
\text { changement climatique } \\
\text { - Réflexion globale sur } \\
\text { l'aménagement du territoire }\end{array}$ \\
\hline \multirow{2}{*}{$\begin{array}{c}\text { Capital social sollicité : } \\
\text { Réseau communautaire et } \\
\text { associatif } \\
\text { Réseau } \\
\text { d'interconnaissance } \\
\text { individuel }\end{array}$} & $\begin{array}{l}\text { Présent mais peu ou pas sollicité } \\
\text { autour des questions climatiques }\end{array}$ & $\begin{array}{c}\text { Très présent et sollicité autour } \\
\text { des questions climatiques }\end{array}$ \\
\hline & $\begin{array}{l}\text { Relation inter-individuelle avec } \\
\text { membre du gouvernement } \\
\text { provincial }\end{array}$ & \\
\hline $\begin{array}{l}\text { Limites de Paction } \\
\text { collective }\end{array}$ & \multicolumn{2}{|c|}{ - Gouvernance locale inadaptée } \\
\hline
\end{tabular}

\subsection{L'adaptation autonome sur le plan collectif}

\subsubsection{Sur le territoire du nord-est}

21 Dans toutes les communautés du nord-est, nous avons constaté l'importante mobilisation du réseau d'entraide pour affronter les tempêtes et réparer ensuite. Les acteurs même vieillissants ont confiance en ce réseau pour les aider à affronter les tempêtes : " Ici t'est jamais tout seul. T'as besoin d'aide tu vas l'avoir, pas financièrement ou quoi que ce soit mais des bras tu vas en avoir tout le temps ».(Répondant Pigeon Hill, 16 février 2012).Par ailleurs, sur 20 questionnaires, 16 répondants affirment pouvoir compter sur leurs proches en cas de tempête. En dehors des épisodes de tempêtes, les communautés sont aussi interpellées par le sort de plusieurs de leurs concitoyens dont les maisons sont continuellement menacées par l'érosion côtière.

\section{Le danger catalyseur d'une action collective?}

À Pigeon Hill, à partir de 2009, des citoyens bénévoles, dont un biologiste retraité, ${ }^{12}$ habitué à travailler avec des ministères, ont pris en charge le problème. Des membres du comité de DSL sont aussi impliqués dans cette démarche. Ils ont mesuré artisanalement le recul de la côte, en ciblant les secteurs les plus problématiques et ont démarché différentes instances gouvernementales pour obtenir un soutien financier et technique. Cette mobilisation se fait dans la continuité de différentes actions locales portées par des organismes locaux comme la paroisse religieuse, les Chevaliers de Colomb, l'Union des pêcheurs des Maritimes ou le comité de gestion du quai autour d'enjeux collectifs comme 
l'entretien de l'église ou l'amélioration des conditions de navigation du havre portuaire. L'objectif du projet est de protéger la côte avec des enrochements, dans une logique de lutte contre l'érosion. Cette solution semble être la seule pour laquelle il leur parait possible d'obtenir des fonds à court terme de la part du gouvernement, hors, pour plusieurs habitations il y a urgence. Dans ce cadre, des ententes sont signées avec les propriétaires riverains qui cèdent leurs droits sur une partie des terrains. Ainsi, en 2014, le ministère du transport du N-B enroche plusieurs terrains afin de protéger la route, et par conséquent certaines habitations situées entre la route et la mer. En l'absence de programme provincial correspondant à la demande de la communauté, l'intervention gouvernementale se fait en utilisant un autre programme, celui visant la protection des routes provinciales, et en l'adaptant ponctuellement. Cette action collective locale a comme objectif de diminuer la vulnérabilité physique des certaines habitations en « attirant » l'attention du gouvernement sur leur sort, sans générer de réflexion globale sur l'aménagement du territoire.

Cependant à l'issu d'un travail de réflexion encadré par l'Institut de recherche sur les zones côtières (IRZC) en 2013, et auxquels des ressortissants Cap-Bateau et Pigeon Hill et du village voisin de la municipalité de Sainte-Marie-Saint-Raphaël ont participés, des recommandations portant sur la réglementation des usages dans les zones les plus à risques ont été faites. Ces recommandations ne sont pas contestées, mais ne seront pas portées localement. Il faut mentionner que la structure organisationnelle des DSL ne facilite pas l'émergence de règlement spécifique à ce territoire car toute modification doit passer par l'échelon provincial. De plus, la population locale est peu habituée aux principes de l'aménagement du territoire qui est y presque inexistant. Ainsi, même si plusieurs répondants mentionnent qu'il n'est pas cohérent de permettre la construction et la consolidation d'habitat dans des zones inondables ou en érosion, la planification et la réglementation des usages du territoire est localement peu populaire : "Le plan vert, là, on n'a plus le droit de loger dans les côtes [...] On a du terrain en masse, à côté, mais t'as plus le droit de faire rien " (Répondant Pigeon Hill, 15 février 2012).

24 Au-delà de la présence d'une menace, la capacité de mobiliser des ressources: intellectuelle, réseau, temps, finance est importante pour qu'il y ait action collective. Dans le cas du DSL de Cap-Bateau, adjacent à PH, plusieurs maisons sont aussi considérées comme « à risque immédiat » mais il n'y pas de mobilisation collective. La petite taille de la communauté, le fait qu'il n'y ait pas d'associations spécifiques à ce village puisque les résidents utilisent le quai et l'église de la municipalité voisine de Sainte-Marie-SaintRaphaël, le faible niveau d'éducation des leaders locaux peuvent expliquer partiellement la faiblesse de l'engagement collectif dans ce cas. Les habitants se sentent aussi délaissés par le gouvernement : « Nous autres on s'adapte mais c'est le gouvernement qui ne s'adapte pas » (répondant Cap-Bateau, 16 février 2012).

\section{La place de la recherche partenariale et des associations environnementales?}

Depuis 2012, PH et CB sont incluses dans un projet d'accompagnement porté par des chercheurs, cependant les liens entre les chercheurs et les acteurs locaux sont encore peu développés. De plus, l'organisme environnemental qui couvre le territoire de l'île Lamèque ne fait pas de sensibilisation aux effets des changements climatiques et aux options d'adaptation et aucun autre organisme n'intervenait en ce sens avant 2012 sur ce territoire. Dans ces communautés, le CC est une réalité : les acteurs locaux sont bien conscients des changements actuels de leur environnement. Mais il n'y a pas eu de 
réflexion globale sur la notion d'adaptation qui est surtout cantonnée aux réponses techniques visant à contrôler la vulnérabilité physique des infrastructures. Par exemple, le fait que le changement climatique amplifie les processus d'érosion est un des arguments utilisés par les leaders de PH, mais leur discours reste centré sur la gestion du risque immédiat aux habitations.

\section{Le capital social : un frein au processus d'adaptation?}

Il existe de nombreux liens familiaux et amicaux unissant les usagers des territoires étudiés qui sont largement mobilisés lors des tempêtes et en "post-tempête » et contribuent ainsi à la capacité d'adaptation de ces communautés. Mais, l'existence de liens forts peut aussi affecter la capacité d'adaptation (Wolf et al., 2010). Lorsque les enjeux ou les solutions apportées semblent menacer la réputation, la survie ou l'unité de la communauté, un discours minimisant ces enjeux peut se développer pour protéger au moins à court terme la communauté (Sfez, 2010 ; .Dans le nord-est des acteurs craignent que la diffusion d'informations alarmistes ne nuise à l'image de leurs villages, notamment au plan du recrutement de nouveaux habitants. Il peut aussi être difficile de porter des approches perçues comme étant contraignantes, telles que un renforcement des règles d'urbanisation sur le littoral ou la municipalisation du territoire ${ }^{13}$, puisqu'il est implicitement admis qu'un membre de la communauté devrait éviter de désavantager les autres membres de la communauté. Un répondant mentionne, par exemple, qu'il serait préférable que la sensibilisation à la protection de l'environnement se fasse par des personnes externes à la communauté : «Ici tout le monde se tient on est un petit village. Fait que, c'est sûr, c'est mieux qu'il y ait un enseignement général de fait, plutôt que de cibler une personne en tant que tel. Parce qu'ici oublie ça, ça ne marchera pas. On va se faire péter nos vitres, pis on va se faire péter nos chars [voitures] » (Répondant Pigeon Hill, 16 février 2012).

\subsubsection{Sud-est du Nouveau-Brunswick}

27 À Cocagne et à Grande-Digue, comme dans le nord-est l'entraide et la mobilisation d'un capital social local caractérise l'adaptation autonome locale. À l'inverse du cas précédent, la présence d'associations environnementales impliquées depuis longtemps sur ce territoire influence la manière dont les communautés se mobilisent.

\section{Le secteur associatif : moteur d'une action collective locale}

Notre enquête de terrain met en évidence la présence d'un secteur associatif fortement développé dans les communautés étudiées. Plus d'une vingtaine d'organismes communautaires sont recensés dans la zone d'étude pour une population totale d'à peine 5000 habitants (Chouinard et al., 2013). Ces associations de tout ordre, Club d'âge d'or, Association de pêcheurs récréatifs, Association de bassin versant, Mouvement local slow Food, Transition Cocagne, Groupe de développement durable, etc. contribuent au maintien d'un capital social dense au sein de ces communautés rurales.

À la différence de la situation exposée précédemment, les questions liées à l'environnement font l'objet de discussion au sein de ces communautés depuis plus de 20 ans. Au départ, ces discussions avaient lieu au sein des comités de développement durable mis en place au début des années 1990 par le gouvernement provincial. Suite au retrait du gouvernement, les personnes engagées dans le comité de développement durable du 
secteur de Cocagne décident de créer une association environnementale afin de poursuivre le travail ${ }^{14}$. Ce qui va donner naissance à un acteur associatif aujourd'hui incontournable dans la communauté : le Groupe de développement durable du pays de Cocagne (GDDPC). Sa mission est de "faciliter l'engagement citoyen pour assurer la viabilité des communautés du bassin versant de Cocagne. $»^{15}$. Le GDDPC regroupe les communautés situées dans le bassin versant de la rivière et de la baie de Cocagne (Grande-Digue, Cocagne, Notre-Dame, Irishtown et Grand Saint-Antoine) et représente un territoire de $400 \mathrm{~km}^{2}$. Il a développé des partenariats diversifiés avec les écoles et les entreprises locales, le gouvernement provincial et fédéral, l'Université de Moncton, des organismes à but non lucratifs, etc. Le GDDPC joue un rôle de stimulateur et de catalyseur à travers son travail de sensibilisation, d'éducation environnementale et d'information. Il organise des réunions d'information sur toutes les thématiques environnementales (biodiversité, gestion des déchets, réchauffement climatique, agriculture locale et biologique, etc.) ainsi que des programmes de sensibilisation dans les écoles, etc. Il constitue pour les habitants de ces communautés une référence pour obtenir de l'information, de l'aide, organiser de la sensibilisation, etc. Il est considéré au niveau communautaire comme un «chien de garde » de l'environnement et du développement durable, mais aussi du changement climatique.

\section{Le changement climatique : un catalyseur de l'action collective?} les préoccupations des résidents ont augmenté. Les résidents estiment que l'aménagement tel qu'il est organisé sur la zone côtière de leur territoire contribue au renforcement de leur vulnérabilité et que de ce fait, l'action du gouvernement n'est pas suffisante. C'est ainsi que la volonté de s'organiser au niveau local afin de mieux gérer les enjeux environnementaux et de développement qui les concernent s'est peu à peu construite. Dans ce cadre, le projet d'élaborer un plan local d'adaptation a émergé. Le président du DSL de Cocagne souligne que sans le GDDPC, aucune action n'aurait été mise en œuvre au sein de la communauté sur la question du climat : «s'il y avait juste le DSL, on serait resté sur notre chaise »(Focus group Cocagne, 30 juillet 2012).

Pour mener à bien l'élaboration de leur plan local d'adaptation, le GDDPC est venu interpeller les chercheurs de l'Université de Moncton afin qu'ils puissent les accompagner dans cette démarche. Un plan préliminaire d'adaptation a été élaboré et intitulé "L'adaptation au changement climatique dans les DSL de Cocagne et de Grande Digue. Vers une planification côtière durable. Brochure d'information pour l'aménagement durable du littoral » (Chouinard et al., 2012). L'objectif de ce document publié en 2012 était de fournir un appui à la prise de décision envers l'adaptation à l'érosion côtière et aux autres impacts du changement climatique dans la baie de Cocagne, concernant en particulier les DSL de Cocagne et Grande-Digue. Ce plan destiné aux propriétaires côtiers et aux membres de la communauté au sens large, s'inscrit dans une démarche instructive et pédagogique. Le contenu de ce plan préliminaire d'adaptation démontre la capacité des 
communautés locales à se positionner et à formuler des solutions d'adaptation au changement climatique. Ces solutions sont d'ailleurs plus élaborées que ce que le gouvernement provincial est en mesure de fournir à ce jour.

\section{Le rôle de la recherche partenariale}

Dans ce processus de mobilisation locale de la communauté de Cocagne, nous avons remarqué que l'université locale a joué un rôle clé d'accompagnateur. L'Université de Moncton travaille en partenariat avec le GDDPC depuis sa création. Un groupe de chercheurs de l'université avait même participé aux réflexions menées dans le cadre de l'élaboration de la vision du GDDPC. Il est évident que dans le cas des communautés étudiées le lien établi entre l'Université de Moncton et le GDDPC a grandement facilité la mobilisation de la communauté autour des problèmes environnementaux et climatiques. Enfin, un rapport portant spécifiquement sur les impacts du CC dans la région du Sud-est, édité en 2006 par Environnement Canada, et auquel ont participé plusieurs des chercheurs de l'Université de Moncton, a largement été utilisé pour sensibiliser et informer les gens de communauté.

\section{Limites et évolution de la gouvernance locale}

Malgré le soutien de l'université régionale, les actions menées par le GDDPC ont une portée relativement circonscrite. En effet, le plan préliminaire réalisé en 2012 se limite à la sphère d'influence du GDDPC, ne s'étend pas à la communauté toute entière et ne peut faire l'objet d'une réelle mise en œuvre. Ce plan ne pourra être effectif qu'à travers la mise en place de certaines réglementations, et pour cela il est nécessaire qu'un conseil d'élus puisse se réunir. De même que pour réguler l'aménagement du territoire en particulier en zone côtière, et mettre en place des arrêtés sur la construction par exemple ou pour instaurer des mesures d'urgence adaptée à leur besoin, une autorité publique locale est nécessaire. Pour les enquêtés, il est en effet indispensable de réguler, gérer la construction en zone côtière, même si, cette dernière a pris beaucoup de valeur ces dernières années, ils ont tout à fait conscience qu'elle contribue aussi à renforcer la vulnérabilité de leur territoire. Un résident, membre également d'un comité de DSL, souligne que :

"Le fait qu'il n'y ait pas de plan d'aménagement à Cocagne est une de nos préoccupations, on peut encore mettre des maisons partout, on perd un peu notre paysage agricole, les terres agricoles. (...) Le fait de ne pas être municipalisé, c'est un des points de nos désavantages, un autre est de pouvoir mieux guider le développement de la côte » (Répondant Cocagne, 31 mai 2012).

En 2014, la communauté de Cocagne s'est dotée d'une autorité locale en devenant une communauté rurale. Cette évolution du processus de gouvernance locale au niveau des communautés étudiées est très positive mais comme le rappelaient de nombreux enquêtés interrogés: "face à la problématique du climat, une stratégie plus régionale est nécessaire pour mieux coordonner et harmoniser les actions menées au niveau local» (Focus Group, Cocagne, juillet 2012), d'où la nécessité d'interactions entre l'échelle locale et provinciale. 


\section{Conclusion}

37 Nous constatons que malgré une occupation du territoire différenciée, avec un littoral moins densément peuplé et une villégiature moins développée au Nord-est, les principaux enjeux d'adaptation sont similaires sur les deux sites. Les habitants cherchent en premier à se protéger et à réduire les risques pour maintenir leurs modes de vie. Dans les deux cas, nous observons que des liens personnels et des interrelations individuelles (voir tableau 2) sont activités, renvoyant au capital social individuel au sens de P. Bourdieu. Comme nous l'avons montré, ces liens forts peuvent aussi générer de l'inertie et constituer une forme de frein à l'adaptation. Malgré ces convergences, nos résultats mettent en évidence des différences majeures entre les deux sites en matière de capacité à agir collectivement:les acteurs impliqués, les liens mobilisés et les finalités de ces mobilisations ne sont pas les mêmes dans les deux zones à l'étude. À PH par exemple, les acteurs se mobilisent en s'appuyant sur des relations formalisées et personnelles, pour obtenir du gouvernement des ressources techniques et financières afin de répondre à un danger immédiat. Alors que, dans le Sud-est, l'emphase est mise davantage sur l'aménagement du territoire. Le développement soutenu et rapide de la villégiature dans ce secteur peut expliquer partiellement cette différence, mais l'intervention importante d'associations environnementales et de la recherche dans le sud-est favorise aussi l'émergence d'une réflexion globale sur l'occupation du territoire. La réponse collective au CC s'appuie alors sur un capital social fondé sur le renforcement des liens entre acteurs et avec des organisations clés. Nous retrouvons ici plusieurs caractéristiques du capital social tel que défini par R. D. Putnam (1999) ou W. N. Adger (2003).

Au nord-est comme au sud-est du Nouveau-Brunswick malgré leurs efforts d'adaptation, les acteurs se heurtent à un défi majeur : le manque de gouvernance locale. Dans les deux cas, l'impact des mobilisations collectives est limité par la manière dont sont administrés les territoires au NB (DSL) et par l'importante centralisation des décisions qui en résulte (Noblet, 2015). Compte tenu de ce contexte, il est impossible, par exemple, d'imposer des restrictions sur le développement domiciliaire en zone côtière ou de soutenir adéquatement les acteurs sectoriels dans leurs efforts de transition. Nous parvenons au même type de conclusion que P. Evans à propos de la synergie entre action publique, capital social et développement. Pour lui, les blocages à l'instauration d'une synergie entre ces trois éléments reposent moins sur un manque de capital social à l'échelle micro mais plus sur la difficulté du capital social à s'étendre, et à générer des liens de solidarité et de l'action sociale à une échelle qui est politiquement et économiquement efficace (Evans, 1996). Pour dépasser ces limites, au sud-est les acteurs visent la municipalisation du territoire afin d'être davantage en mesure d'intégrer les efforts d'adaptation portés de manière autonome localement à l'action publique en la matière. Au Nord, nous observons l'émergence d'une action à l'échelle régionale. En effet, des acteurs de toute la Péninsule acadienne collaborent depuis 2012 à l'élaboration d'une approche d'adaptation regroupant les différentes communautés de la région. Ce collectif permet de créer des liens entre les communautés et avec les chercheurs et les organisations environnementales régionales et de mobiliser du soutien logistique et financier au niveau du gouvernement. Il vise une planification concertée et anticipée de l'adaptation et non pas réactive et au service d'intérêts particuliers ou partisans. 
Enfin, ces efforts menés à l'échelle locale ont besoin pour se renforcer d'une plus grande intervention du gouvernement provincial. En effet, des cadres d'actions provinciaux, assortis de moyens et d'une volonté politique affichée, encourageraient la mise en œuvre de démarches locales comme la relocalisation ou l'adaptation des règlements en les intégrant à un processus d'adaptation généralisé à l'échelle de la province.

\section{BIBLIOGRAPHIE}

Adger, W. N., 2000, “Social and ecological resilience: are they related?", Progress in Human Geography, 24, 3, p. 347-364.

Adger W. N., 2003, "Social Capital, Collective Action, and Adaptation to Climate Change", Economic Geography, 79, p. 387-404.

Adger W. N. et al., 2003, "Adaptation to Climate Change in the Developing World", Progress in Development Studies, 3, p. 179-195.

Aubé M. et B. Kocyla, 2012, Adaptation aux changements climatiques : planification de l'utilisation du territoire à Shippagan, Le Goulet et Bas-Caraquet, Institut de recherche sur les zones côtières inc./ Commission Aménagement de Péninsule Acadienne, Fonds Fiducie de l'Environnement.

Bassett T. J., Fogelman C., 2013, "Déjà vu or something new? The adaptation concept in the climate change literature", Geoforum, 48, p 42-53.

Berkes F., Folke. C., 1998, Linking Social and Ecological Systems: Management Practices and Social Mechanisms for Building Resilience, Cambridge University Press, New York, 476 p.

Bernatchez P., Fraser C. Friesinger, S., Jolivet, Y., Dugas, S., Drejza, S. et Morissette, A., 2008, Sensibilité des côtes et vulnérabilité des communautés du golfe du Saint-Laurent aux impacts des changements climatiques. Laboratoire de dynamique et de gestion intégrée des zones côtières, UQAR. Rapport de recherche remis au Consortium OURANOS et au FACC, $256 \mathrm{p}$.

Bourdieu P., 1980, « Le capital social », Actes de la recherche en sciences sociales, 31, 1, 1980, p. 2-3.

Callois J.-M., 2006,"Les relations sociales frein ou moteur de la durabilité ; approche par la notion de rayon de confiance ", Développement durable et territoires, Dossier 8, 3, connexion le 29 Juin 2015, http://developpementdurable.revues.org/3284.

Caron D. 2014, « Opposition grandissante au projet de regroupement sur les îles Lamèque et Miscou », Acadie Nouvelle. 6 février 2014.

Chouinard O., Koné A., 2013, Dundas, Grande-Digue, Cocagne, Profil des communautés, N- B, Université de Moncton, ARUC-DCC.

Chouinard O., Tartibu N., Gauvin J., 2012, Adaptation réfléchie en milieu côtier : démarche d'engagement avec les résidents du milieu côtier des DSL de Cocagne et de Grande Digue, par le Programme de Maitrise en Etude de l'environnement, Université de Moncton, pour le Fonds de Fiducie en environnement du Nouveau-Brunswick, 2012.

Coleman J. S., 1998, "Social Capital in the Creation of Human Capital", American Journal of Sociology , 94, p. 95-121. 
Daigle R., 2014, Updated Sea-Level Rise and Flooding Estimates for New Brunswick Coastal Sections, Based on IPCC $5^{\text {th }}$ Assessment Report. R.J. Daigle Enviro pour New Brunswick Department of Environment, Climate Change Secretariat.

Environnement Canada, 2006, Impact de l'élévation du niveau de la mer et du changement climatique sur la zone côtière du sud-est du Nouveau-Brunswick, Environnement Canada, 646 p.

Evans P., 1996, "Government Action, Social Capital and Development: Reviewing the Evidence on Synergy", World Development, 24, p. 1119-1132.

Finn J-G, 2008, Bâtir des gouvernements locaux et des régions viables : plan d'action pour l'avenir de la gouvernance locale au Nouveau-Brunswick, Rapport du commissaire sur l'avenir de la gouvernance locale.

Fontan J-M., 2010, « Recherche partenariale en économie sociale : analyse d'une expérience novatrice de coproduction des connaissances ", La Revue de l'innovation dans le secteur public, 15, 3.

Guillemot et al., 2013, « La perception du risque et l'engagement dans des stratégies d'adaptation aux changements climatiques dans deux communautés côtières de la péninsule acadienne ", Vertigo la revue électronique en sciences de l'environnement, 14, https://vertigo.revues.org/15164.

Hébert C. Aubé M., 2015, Relocalisation à Sainte-Marie-Saint-Raphaël, Cap-Bateau et Pigeon Hill, Institut de Recherche sur les Zones Côtières/Fonds en Fiducie pour l'environnement du Nouveau-Brunswick, 49 p.

Jones N., et al., 2012, "Social risk assessment and social capital: A significant parameter for the formation of climate change policies", The Social Science Journal, 49, p. 33-41.

Klein R. J. T, et al., 2007, "Portfolio Screening to Support the Mainstreaming of Adaptation to Climate Change into Development Assistance", Climatic Change, 84 (1), p. 23-44.

Lane D.E., 2015, “Chapter 1, Introduction" in Sebastian Weissenberger and Omer Chouinard, Adaptation to Climate Change and Sea Level Rise: The Case Study of Coastal Communities in New Brunswick, Canada. Springer Briefs in Environmental Science, Springer.

Larsen R. K., et al., 2012, "A framework for facilitating dialogue between policy planners and local climate change adaptation professionals: Cases from Sweden, Canada and Indonesia", Environmental Science and Policy, 23, p. 12-23.

Moser S. C., Ekstrom J. A., 2010, “A framework to diagnose barriers to climate change adaptation" , PNAS, December 21, vol. 107, no. 51, p. 22026-22031.

Noblet M., 2015, L'adaptation au changement climatique en zone côtière au Canada et au Sénégal, une comparaison Nord/Sud. Thèse de doctorat, Science politique, Université Picardie Jules Verne, Amiens, 412p.

Ostrom E., 1996, "Crossing the Great Divide: Coproduction, Synergy, and Development", World Development, 24, p. 1073-1087.

Ostrom E., 2008, Governing the Commons: The Evolution of Institutions for Collective Action, Cambridge University Press, (22 ${ }^{\text {nd }}$ printing), $280 \mathrm{p}$.

Ostrom, E., 2009, A polycentric approach for coping with climate change, Policy Research Working Paper 5095, World Bank.

Ostrom E., 2010, "Polycentric Systems for Coping with Collective Action and Global Environmental Change", Global Environmental Change, 20th Anniversary Special Issue, 20, 2010, p. 550-557.

Pelling M. C., High C., 2005, "Understanding adaptation: What can social capital offer assessments of adaptive capacity ?" Global Environmental Change, 15, p. 308-319. 
Portes A., 1998, "Social Capital: Its Origins and Applications in Modern Sociology", Annual Review of Sociology, 24, p. 1-24.

Putnam R. D., et al., 1994, Making Democracy Work: Civic Traditions in Modern Italy, Princeton University Press.

Putnam R. D., 1999, « Le déclin du capital social aux États-Unis", Lien social et Politiques, $n^{\circ} 41$.

Robichaud A., et al., 2012, Érosion et infrastructures à risque à Sainte-Marie-Saint-Raphaël, péninsule Acadienne, Nouveau-Brunswick. Solutions d'adaptation aux changements climatiques pour l'Atlantique, http://atlanticadaptation.ca/node/332

Sfez L., 2010, « Voyages en France : recherches sur l'adaptation au réchauffement climatique. » Quaderni, 71, Hiver 2009-2010.

Stervinou V. E. et al., 2013, « La perception des changements environnementaux : le cas de la collectivité côtière de Shippagan, Nouveau-Brunswick ", Vertigo la revue électronique en sciences de l'environnement, 13 (1), http://vertigo.revues.org/13482

Wamsler C., Brink. E., 2014, "Interfacing citizens and institutions practice and responsibilities for climate change adaptation", Urban climate, 7, p. 64-91.

Wise R. M., et al., 2014, "Reconceptualising adaptation to climate change as part of pathways of change and response", Global Environmental Change, 28, p. 325-336.

Wolf J., et al., 2010, "Social Capital, Individual Responses to Heat Waves and Climate Change Adaptation: An Empirical Study of Two UK Cities", Global Environmental Change, 20, p. 44-52.

Woolcock M., 1998, "Social Capital and Economic Development: Toward a Theoretical Synthesis and Policy Framework", Theory and Society, 27 (2), p. 151-208.

Woolcock M., Deepa N., 2000, "Social Capital: Implications for Development Theory, Research, and Policy", The World Bank Research Observer, 15, p. 225-249.

\section{NOTES}

1. W. N. Adger s'appuie notamment sur les travaux de M. Woolcock $(1998,2000)$ et de D. Narayan (2000) pour qui le capital social renvoie aux normes et réseaux permettant aux individus d'agir collectivement.

2. 1700 habitants des DSL des deux îles ont signé une pétition, contre le projet de regroupement entre les DSL et les municipalités de l'île. Le président du DSL de Cap-Bateau est un des leaders de ce mouvement (Caron, 2014, p. 6).

3. Par exemple à Cap-Bateau, sur les 20 questionnaires appliqués de part et d'autre de la route longeant la mer : 1 seule maison est une résidence estivale, 3 maisons ont 100 ans ou plus et 11 maisons ont entre 31 à 100 ans, une seule habitation à moins de 10 ans, car elle a été reconstruite suite à un incendie.

4. Coup d'œil sur ma région: http://www.csnb.ca/sites/default/files/documents/ coup_d_oeil_sur_ma_communaute-6-region_de_shippagan_lameque_inkerman-csnb-2014.pdf

5. Selon statistiques Canada, pour l'unité de recensement de Dundas (incluant Cocagne et Grande-Digue) le revenu médian 2006 de toutes les familles de recensement est de 50736 \$ alors qu'il est 41222 \$ pour la Paroisse de Shippagan (incluant PH et CB). Voir aussi les profils « coup d'œil sur ma communauté » http://www.csnb.ca/sites/default/files/documents/ coup_d_oeil_sur_ma_communaute-12-region_de_shediac_saint-antoine_beaubassin-estcsnb-2014.pdf 
6. Ces travaux de recherche s'inscrivaient dans le cadre plus global d'une thèse de doctorat (Noblet, 2015).

7. Profils de personnes interrogées: 1 aquaculteur, 1 propriétaire de gîte, 3 pêcheurs, 1 gestionnaire de projets environnementaux, 2 biologistes (dont 1 responsable d'association environnementale), 2 agriculteurs, 1 administrateur d'une coopérative agricole/membre du comite de DSL, 2 entrepreneurs locaux (dont le gestionnaire de la marina), 2 ouvriers, 1 gestionnaire (usine de transformation du poisson), 2 retraités dont le président du DSL de Grande Digue, 1 horticulteur, 2 employées.

8. Profils de personnes participants au focus group: 1 responsable d'association environnementale, 1 employée, 1 ouvrière, 1 retraité/représentant du DSL de Grande Digue, 1 administrateur de coopérative/membre du comite de DSL, 1 représentant du DSL de Cocagne.

9. Profils de personnes interrogées : 2 pêcheurs, 1 consultant, 4 retraités et 1 employé du secteur de la santé (dont 2 représentants de DSL de Cap-Bateau et 2 représentants DSL de Pigeon Hill), 1 urbaniste de la commission régionale et 1 représentant du gouvernement provincial pour les DSL.

10. À Cap-Bateau, lorsque questionnées sur les raisons de leur attachement à leur maison 15 personnes interrogées sur 20 y associent le fait d'y avoir passé la majorité de leur vie et 12 la proximité (distance à pied) de la famille et des amis.

11. À Pigeon Hill, trois résidences principales ont été déjà déplacées en 2000, 2004 et 2012 et une résidence secondaire en 2011 (Source : Vaillancourt, 2012). À Cap-Bateau, malgré la situation préoccupante de certaines maisons, il n'y a pas eu de déménagement de cette nature récemment. Par contre, une portion entière du village (une route et au moins 8 maisons) a été déménagée en retrait entre 1946 et 1970, à cause de l'érosion graduelle du littoral (communications personnelles, été 2013).

12. Cette personne a un bagage professionnel différent des principaux leaders de la communauté : expérience du gouvernement, scolarité post-secondaire, lui donnant un avantage pour structurer une base de données et des démarches auprès du gouvernement.

13. Cette municipalisation n'impliquerait en effet pas seulement la possibilité d'élire des représentants et de réglementer localement les usages mais aussi une augmentation probable des taxes foncières qui sont moins élevées dans les territoires non municipalisés faiblement pourvus en services.

14. Information issue d'un entretien personnel mené auprès de la directrice du GDDPC, 14 avril 2014.

15. Site internet du GDDPC : http://www.ecopaysdecocagne.ca/index.php?lang=fr 16. Site internet du GDDPC: http://www.ecopaysdecocagne.ca/index.php? option=com_content\&view=article\&id=3\&Itemid=28\&lang=fr

\section{RÉSUMÉS}

Cet article porte sur le rôle du capital social dans le cadre de la mise en œuvre de stratégies d'adaptation au changement climatique dans le secteur du bâti (habitation humaine) en zone côtière. Nos travaux portent sur deux études de cas situées au Nord (Pigeon Hill et Cap-Bateau) et au Sud (Cocagne et Grande Digue) du Nouveau-Brunswick (Canada). Nous constatons que les mobilisations visant la mise en œuvre de stratégies d'adaptation reposent effectivement sur du 
capital social tels que des relations de parenté ou des liens associatifs. Cependant, ces mobilisations ont une portée relativement restreinte étant donné que la gouvernance locale reste faiblement développée au Nouveau-Brunswick. Afin d'améliorer la prise en charge au sein des communautés des enjeux du changement climatique, il serait nécessaire de repenser la synergie entre les échelles et les modes d'action. Ceci permettrait au capital social - indispensable au développement de ces processus locaux d'adaptation - de s'appuyer sur des ressources et des structures supra-locales.

adaptation, changement climatique, capital social, action collective, zone côtière

This article focuses on the role of social capital in the context of the implementation of adaptation strategies to climate change in the building sector in coastal areas. Our work focuses on two case studies located in the North (Pigeon Hill and Cap Bateau) and South (Cocagne and Grande Digue) New Brunswick (Canada). We observe that mobilizations are based on social capital such as family ties or associative links. However, the scope of these mobilizations remains relatively limited because of the weakness of the local governance process in New Brunswick. In order to develop a real capacity for local action, it would be necessary to rethink the synergy between the scales and modes of action. This would allow the social capital -necessary to the local adaptation process- to rely on supra-local resources and structures.

\section{INDEX}

Keywords : adaptation, climate change, social capital, collective action

\section{AUTEURS}

\section{MÉLINDA NOBLET}

Mélinda Noblet est politiste de formation, membre du CURAPP, elle réalise actuellement un postdoctorat au sein de l'Université du Québec à Rimouski et de l'Institut national de santé publique du Québec. Ses thématiques de recherche portent sur la vulnérabilité, l'adaptation aux changements climatiques, la résilience, l'analyse comparée de l'action publique et les rapports Nord/Sud, melinda.noblet@gmail.com

\section{JULIE GUILLEMOT}

Julie Guillemot est docteur en développement régional (UQAR, Rimouski), elle est professeure à l'Université de Moncton (Développement Durable et Zone Côtière). Elle s'intéresse à la gouvernance locale et aux conflits d'usage, julie.guillemot@umoncton.ca

\section{OMER CHOUINARD}

Omer Chouinard est professeur au département de sociologie et au programme de la Maîtrise en études de l'environnement de l'Université de Moncton. Il est co-éditeur d'Adaptation to Climate Change and Sea Level Rise : The Case Study of Coastal Communities in New Brunswick, Canada, Springer Briefs (2015) et de Coastal Zones : Solutions for the $21^{\text {st }}$ Century, Elsevier (2015), omer.chouinard@umoncton.ca 